\title{
1 Evaluation of porcine stem cells competence for somatic cell 2 nuclear transfer and production of cloned animals
}

Jan O. Secher ${ }^{\mathrm{a}, 1}$, Ying Liu ${ }^{2}$, Stoyan Petkov ${ }^{3}$, Yonglun Luo ${ }^{4}$, Dong Li ${ }^{6}$, Vanessa J. Hall ${ }^{6}$; Mette Schmidt $^{1}$, Henrik Callesen ${ }^{2}$, Jacob F. Bentzon ${ }^{5}$, Charlotte B. Sørensen ${ }^{5}$, Kristine K Freude ${ }^{6}$, Poul Hyttel $^{\mathrm{a}, 6}$

a: Corresponding authors: Jan O. Secher (jbsecher@sund.ku.dk) \& Poul Hyttel (poh@sund.ku.dk) 1: Veterinary Reproduction and Obstetrics, Department of Large Animal Sciences, University of Copenhagen, DK1870 Frederiksberg C, Denmark

2: Department of Animal Science, Aarhus University, DK8830 Tjele, Denmark

3: Institute for Farm Animal Genetics (FLI), Neustadt, Germany

4: Department of Biomedicine, Aarhus University, DK8000 Aarhus C, Denmark

5: Department of Clinical Medicine, Aarhus University, DK8200 Aarhus N, Denmark

6: Department of Veterinary Clinical and Animal Sciences, University of Copenhagen, DK1870 Frederiksberg C, Denmark

\section{Abstract} 0

Porcine somatic cell nuclear transfer (SCNT) has been used extensively to create genetically modified pigs, but the efficiency of the methodology is still low. It has been hypothesized that pluripotent or multipotent stem cells might result in increased SCNT efficacy as these cells are closer than somatic cells to the epigenetic state found in the blastomeres and therefore need less reprogramming. Our group has worked with porcine SCNT during the last 20 years and here we describe our experience with SCNT of 3 different stem cell lines. The porcine stem cells used were: Induced pluripotent stem cells (iPSCs) created by lentiviral doxycycline-dependent reprogramming and cultered with a GSK3 $\beta$ - and MEK-inhibitor (2i) and leukemia inhibitor factor (LIF) ( $2 \mathrm{i}$ LIF 9 DOX-iPSCs), iPSCs created by a plasmid-based reprogramming and cultured with $2 \mathrm{i}$ and fibroblast growth factor (FGF) (2i FGF Pl-iPSCs) and embryonic germ cells (EGCs), which have earlier been characterized as being multipotent. The SCNT efficiencies of these stem cell lines were compared with that of the two fibroblast cell lines from which the iPSC lines were derived. The blastocyst 
rates for the $2 \mathrm{i}$ LIF DOX-iPSCs were $14.7 \%$, for the $2 \mathrm{i}$ FGF Pl-iPSC $10.1 \%$, and for the EGCs $34.5 \%$ compared with the fibroblast lines yielding $36.7 \%$ and $25.2 \%$. The fibroblast- and EGCderived embryos were used for embryo transfer and produced live offspring at similar low rates of efficiency (3.2 and 4.0\%, respectively) and with several instances of malformations. In conclusion, potentially pluripotent porcine stem cells resulted in lower rates of embryonic development upon SCNT than multipotent stem cells and differentiated somatic cells.

\section{Key Words}

Pluripotency, induced pluripotent stem cells, embryonic germ cell, transgenic pigs, somatic cell nuclear transfer, handmade cloning. further (Schmidt et al., 2015, 2010).

Earlier studies have indicated that cells derived from early embryos result in increased developmental rates following SCNT (Oback, 2009). Thus, it was hypothesized that the use of pluripotent stem cells (PSCs) for SCNT would result in higher efficiencies and less abnormalities. Considerable efforts have been invested in deriving PSCs in the large domestic species including the pig, which is generally considered to be a valid model for humans due to physiological and anatomical similarities (Bassols et al., 2014; Lelovas et al., 2014). It has, however, proven to be a great challenge to maintain bona fide porcine embryonic stem cells (ESCs) and also the quest for derivation of porcine iPSCs has been associated with several complications including lack of 
transgene silencing and incomplete reprogramming and activation of the endogenous pluripotency network (reviewed by (Telugu et al., 2010)). Some of the problems with piPSC have been sought explained by comparing piPSC to naïve and primed murine embryonic stem cells (mESC). Naïve mESCs can be isolated from the murine ICM just before implantation and depend on leukemia inhibitor factor (LIF) and MEK- and GSK3 $\beta$-inhibitors (2i) in vitro (Nichols and Smith, 2009) whereas primed murine ESCs can be isolated from the murine epiblast just after implantation and can be maintained in vitro by addition of bFGF to the media (Nichols and Smith, 2009). Only the naïve mESC can contribute to germline chimeras and they yield a higher efficiency of SCNT than primed mESC (Nichols and Smith, 2009). The first piPSCs generated (Esteban et al., 2009; Ezashi et al., 2009; Wu et al., 2009) resemble the primed type mESCs in regards to colony morphology and dependence on FGF. Subsequently, several groups have established piPSCs and cultured them supplemented with LIF and $2 \mathrm{i}$ to mimic the conditions which resulted in naïve murine ESCs (Cheng et al., 2012; Fujishiro et al., 2013; Telugu et al., 2011) and in cattle this has resulted in promising results (Kawaguchi et al., 2015). The use of both naïve-like and primed-like piPSCs for SCNT has been reported (Du et al., 2015; Fan et al., 2013; Yuan et al., 2014). Du et al. used naïve-like porcine iPSCs reprogrammed with a non-integrating episomal vector and cultured with leukemia inhibitory factor (LIF) as well as GSK3 $\beta$ - and MEK-inhibitors (2i) but was unable to produce live piglets following SCNT (Du et al., 2015). Similar results were obtained by Fan et al. who used primed-like iPSCs reprogrammed with a doxycycline regulated lentiviral construct and cultured with fibroblast growth factor (FGF) (Fan et al., 2013). Interestingly, Fan and co-workers also found that if they submitted the iPSCs to spontaneous differentiation prior to SCNT, they were able to achieve live piglets. This is in contrast to the results obtained in mice where the SCNT efficiency has been demonstrated to increase with differentiation potency of the cells used as nuclear donors (Nichols and Smith, 2009; Okita et al., 2011).

Here, we provide a description of our experience with SCNT using a transgenic male Large White/ Landrace perinatal fibroblast line (WL-pNFs) expressing the Venus (green fluorescence) protein, a iPSC line derived from the WL-pNFs using a doxycycline regulated lentiviral construct and cultured with $2 \mathrm{i}$ and LIF (2i LIF DOX-iPSCs), a male wild type Göttingen embryonic fibroblast line (G-PEFs), a iPSC line derived from the G-PEFs (2i FGF Pl-iPSC) using the non-integrating episomal method (Okita et al., 2011) and cultured with FGF, and finally a Yucatan female transgenic porcine embryonic germ cell line (EGC) overexpressing the human wildtype PCSK9 (for an overview over the experiments and the cell lines please see Figure 1A-C and 1D). We compare 
initial embryonic development and results from embryo transfer to the hypothesis that the capacity of porcine cells to sustain embryonic development upon SCNT is positively correlated with their developmental potency, i.e. that PSCs are more capable of being reprogrammed than somatic cells and that multipotent stem cells might have an intermediate capacity.

\section{Material and methods}

\subsection{Cells and iPSC reprogramming}

Large White/Landrace porcine neonatal fibroblasts (WL-PNFs) carrying a transgene encoding the green fluorescent protein Venus with a constitutive promotor (Garrels et al., 2011) were kindly provided by Wilfried Kues and cultured in high-glucose Dulbecco's modified Eagle's medium (DMEM AQ; Sigma-Aldrich, St. Louis, MO, USA) supplemented with 1\% Penicillin/Streptomycin (Pen/Strep; Sigma Aldrich) and 10\% fetal bovine serum (FBS; Hyclone, GE Health Care Life Sciences, Chicargo, IL, USA). The WL-PNFs were reprogrammed using a lentiviral construct as described previously (Secher et al., 2015). In brief, a doxycycline-regulated CAG promoter containing porcine $O C T 4, c-M Y C$, SOX2 and KLF4 was used for lentiviral reprogramming of WLPNFs into iPSCs (Nethercott et al., 2011). The resulting iPSC (2i LIF DOX-iPSCs) were maintained on mitomycin-C-treated mouse embryonic fibroblasts (MEFs) in DMEM/F12 medium

115 (Sigma Aldrich) supplemented with 20\% KnockOut Serum Replacement (Invitrogen, Waltham, 116 MA, USA ), 1x Pen/Strep (Sigma Aldrich), 1x nonessential amino acids (Sigma Aldrich), $100 \mu \mathrm{M}$ 117 b-mercaptoethanol (Life Technologies), $10 \mathrm{ng} / \mathrm{mL}$ leukemia inhibitory factor (LIF) (Millipore, 118 Billerica, MA, USA), two kinase inhibitors (1 $\mu$ M PD0325901 [Sigma Aldrich], $3 \mu$ M CHIR99021 119 [Sigma Aldrich]), and $2 \mu \mathrm{g} / \mathrm{mL}$ doxycycline (Sigma Aldrich) (piPSC medium). Cells were 120 dissociated with 1x TrypLE (Gibco, Waltham, MA, USA ) and passaged 1:6 every 7 days. Please 121 notice that this cell line differs from the iPSC line reprogrammed with a doxycycline regulated 122 Lentivirus construct made by Fan et al. (Fan et al., 2013) and used for their SCNT experiments by 123 the fact that we used $2 \mathrm{i}$ LIF culture conditions and they used FGF. A full characterization of the $2 \mathrm{i}$ LIF DOX-iPSC is in press (Secher et al., 2017).

Göttingen porcine embryonic fibroblasts (G-PEFs) obtained from embryos at day 25 of gestation were reprogrammed and characterized. In brief, electroporation-based delivery of episomal plasmids (Okita et al., 2011)) purchased from Addgene Inc. (Cambridge, MA), pCXLE-hOCT3/4- 
shp53 (Addgene plasmid \#27077), pCXLE-hSK (Addgene plasmid \#27078), and $p C X L E-h U L$

130 (Addgene plasmid \#27080) were used. The resulting plasmid-based iPSCs (2i FGF Pl-iPSCs) were 131 maintained on mitomycin-C-treated MEFs in DMEM/F12 medium (Sigma Aldrich) supplemented 132 with 20\% KnockOut Serum Replacement (Invitrogen), 1x Pen/Strep (Sigma Aldrich), 1x 133 nonessential amino acids (Sigma Aldrich), $100 \mu \mathrm{M}$ b-mercaptoethanol (Life Technologies), 20 $134 \mathrm{ng} / \mathrm{mL}$ human recombinant basic fibroblast growth factor (bFGF) (Prospec, East Brunswick, NJ), 135 and two kinase inhibitors; $1 \mu \mathrm{M}$ PD0325901 (Sigma-Aldrich), which inhibits MEK signal pathway, 136 and $3 \mu \mathrm{M}$ CHIR99021 (Sigma-Aldrich), a GSK3 $\beta$ inhibitor. Cells were dissociated with 1x TrypLE (Gibco) and passaged 1:6 every 4-5 days. Please notice that this cell line differs from the iPSC line reprogrammed with an episomal plasmid made by Du et al. (Du et al., 2015) and used for their SCNT experiments by the fact that we used 2i FGF culture conditions and Du et al. used the 2i LIF culture condtions. A full characterization of the 2i FGF Pl-iPSC is pending and will be submitted later this year ( $\mathrm{Li}$ et al., manuscript in preparation).

Yucatan mini-pig EGCs were isolated from individual embryos, maintained, and characterized as described previously (Petkov et al., 2011). Prior to SCNT, the EGCs were genetically modified using a Sleeping Beauty transposon system for insertion of human wildtype PCSK9 comprising a FLAG-tag as described previously (Al-Mashhadi et al., 2013) with the aim of creating a large animal model for hypercholesterolemia and atherosclerosis. The vector pSBT/ApoEHCR-hAATpWT-PCSK9-FLAG, comprising the apoE locus control region (HCR) and the human $\alpha 1$-antitrypsin promoter (hAAT), was constructed in essentially the same way as previously described for pSBT/ApoEHCR-hAATp-D374Y-PCSK9 (Al-Mashhadi et al., 2013) except that the human WT PCSK9 cDNA comprising a FLAG-tag was inserted into the vector backbone instead of the mutant D374Y form of human PCSK9. Briefly, EGCs were seeded in six-well plates at a density of $3 \times 10^{5}$ cells per well and, on the next day, transfected with $1.0 \mu \mathrm{g}$ of pSBT/ApoEHCR-hAATp-WTPCSK9-FLAG plasmid and $20 \mathrm{ng}$ of pCMV-SB100X (encoding the hyperactive SB100X transposase) using Effectene Transfection Reagent (Qiagen, Hilden, Germany). Three days posttransfection, selection with puromycin $(1.0 \mu \mathrm{g} / \mathrm{ml})$ was initiated and maintained for 12 days. Puromycin-resistant colonies were subsequently isolated, expanded, and pooled prior to being used 


\subsection{Somatic cell nuclear transfer (SCNT)}

All cells for SCNT were grown to reach to full confluence and the media was not changed by the EGC for the last 3 days before SCNT to force as many cells as possible into the G1 or G2 phase. The stem cell colonies were controlled for changes in colony morphology as a sign of beginning differentiation, before they were used for SCNT; this revealed that it not was possible to starve the iPSC, and therefore both iPSC and fibroblasts had their media changed every day up to the SCNT to make them comparable. SCNT by handmade cloning was performed as described previously ( $\mathrm{Li}$ et al., 2013) using donor cells of different developmental potency ranging from fibroblasts (WLPNFs) over EGCs to iPSCs (2i FGF Pl-iPSCs and 2i LIF DOX-iPSCs). The cell lines were from polyclonal origin and at least two replicates were made for each cell line. Reconstructed embryos created from G-PEF, WL-PNF, EGC and 2i FGF Pl-iPSC donor cells were cultured in PZM3 (Li et al., 2013) until day 5 to 6 after activation. Reconstructed embryos created from 2i LIF DOX-iPSC were cultured in PZM3 until 4 days after activation followed by division into 3 groups. Group 1 was cultured in PZM3 from day 4 to day 6; Group 2 was cultured in PZM3 with 10\% fetal calf serum (FCS) (Sigma-Aldrich F7524) from day 4 to day 6; Group 3 was cultured in PZM3 with $2 \mu \mathrm{g} / \mathrm{ml}$ doxycycline (Sigma Aldrich, Cat. \# D9891) from day 4 to day 6 . At day 6 all embryos were evaluated for development and quality prior to embryo transfer. SCNT-derived morulae and blastocysts derived from WL-PNFs and EGCs were collected on days 5 and 6 prior to embryo transfer. Blastocyst rates (BR) were compared between donor cell types and culture conditions using the logistic regression function in SAS 9.3. Furthermore, the embryos and piglets derived from the WL-PNFs and 2i LIF DOX-iPSCs were evaluated for Venus fluorescence expression (green fluorescence).

\subsection{Embryo transfer}

General animal welfare guidance was followed and permission for transfer of the cloned embryos was granted by the Danish Animal Experiments Inspectorate (2004-561-925; 2013-15-2934-00943). Embryo transfer was performed surgically to surrogate sows as described previously (Schmidt et al., 2010, 2011) using reconstructed embryos from WL-PNF and EGC donor cells. 
192 Ear biopsies were taken from all the cloned EGC-derived piglets at birth in order to isolate genomic 193 DNA for molecular genetic analyses. The cloned piglets were genotyped by PCR for the presence 194 of the WT-PCSK9-FLAG transgene using a Pfx Platinum polymerase (Thermo Fischer Scientific), 195 the forward hAAT-specific primer 5'- GGATCTTGCTACCAGTGGAAC-3', the reverse FLAG196 specific primer 5'- ACTTGTCATCGTCGTCCTTG-3', and the PCR protocol: 1 cycle of $94^{\circ} \mathrm{C}$ for 1972 mins, 35 cycles of $94^{\circ} \mathrm{C}, 20$ secs, $55^{\circ} \mathrm{C}$ for 30 secs, $68^{\circ} \mathrm{C}$ for 30 secs, followed by 1 cycle of $68^{\circ} \mathrm{C}$ 198 for 7 mins.

200 Genomic DNA isolated from the cloned EGC-derived animals was analyzed by Southern blotting. 201 Genomic DNA (15 $\mu \mathrm{g})$ was digested overnight with SpeI, which cleaves at a single site within the transposon. The digested DNA was electrophoresed in a $0.8 \%$ agarose gel and transferred to a Hybond membrane (GE Healthcare, Chicargo, IL, USA). The membrane was hybridized overnight using a $[\alpha-32 \mathrm{P}]-\mathrm{dCTP}-$ labeled probe specific for the puromycin resistance gene in the vector. The surviving founder animal was subsequently bred with a wildtype Yucatan boar to generate F1 progeny. Genomic DNA isolated from all offspring was subjected to analysis by Southern blotting

The surviving EGC-derived founder animal was karyotyped using Q-banding and the software Quips Karytyper version 3.1 (Vysis). Briefly, fibroblasts were isolated from the cloned piglet and 211 cultured in slideflasks. The cells were arrested in metaphase by addition of colcemid and subjected 212 to fixation and staining with DAPI prior to analysis. The DOX-iPSCs and Pl-iPSCs were submitted 213 to karyotyping at Cell Guidance Systems (Babraham Research Campus, Cambridge, UK).

215 The EGC-derived founder animal was euthanized at approximately 2.5 years of age and various 216 tissues were isolated and snap-frozen for quantative PCR analysis. RNA was isolated using an 217 RNeasy kit (Qiagen). One $\mu$ g RNA was used for cDNA synthesis employing a RevertAid First 218 Strand cDNA Synthesis kit (Fermentas, Waltham, MA, USA ). Quantative PCR was performed 219 using Maxima SYBR Green qPCR Master Mix (Fermentas) and primers specific for human PCSK9 220 (forward primer: 5'-ACCATGGGCACCGTCAGCTC-3', reverse primer: 5'221 AAGGTGGCTGTGGTTCCGTG-3'). The porcine $\beta$-actin gene ACTB was used as reference gene 222 (forward primer: 5'-CACGCCATCCTGCGTCTGGA-3', reverse primer: 5'- 


\section{Results}

\subsection{Blastocyst rates and quality}

230 The SCNT experiments are summarized in Figure 1 and the results and statistical analysis are 231 summarized in Table 1A \& 1B. SCNT based on WL-PNFs as donor cells resulted in a high 232 blastocyst rate $(36.7 \%, \mathrm{n}=392)$ and good blastocyst quality (Figure 2A \& 2F). SCNT based on G233 PEFs yielded a significantly lower blastocyst rate $(25.2 \%, \mathrm{n}=190)$ and impaired quality (Figure 2B) 234 compared to the WL-PNFs ( $\mathrm{p}=0.006)$. The blastocyst rate resulting from SCNT with EGC (34.5\%, $235 \mathrm{n}=148$ ) was not statistically different from those obtained from the WL-PNFs and the G-PEFs. 236 SCNT based on 2i LIF DOX-iPSC cultured in PZM (Group 1) resulted in significantly lower 237 blastocyst rate $(10.7 \%, \mathrm{n}=122)$ and low quality (small blastocysts with uneven outline and loose 238 blastomeres in the perivitelline space; Figure 2C, H). This low developmental capacity corresponds to earlier reported results following iPSC-derived SCNT (Du et al., 2015; Fan et al., 2013). Since we have experienced a positive effect of culturing porcine iPSC chimeric embryos in PZM3 with $10 \%$ FCS, we subsequently cultured the iPSC-derived reconstructed embryos in PZM3 with 10\% FCS (Group 2), which, however, did not statistically significant improve the blastocyst rate $(20.6 \%$ $\mathrm{n}=63, \mathrm{p}=0.069$ ) nor quality (Figure 2D, I). Also, we added doxycycline to the PZM3 4 days after activation of the reconstructed embryos (Group 3) in order to secure the expression of the pluripotency genes during formation of the ICM (Cao et al., 2014). Again, this did not improve the blastocyst rate $(22,2 \%, n=18, p=0.171)$ whereas the blastocyst quality was improved (Figure 2E, J). Due to the very low blastocyst quality in general, we did not attempt transfer of the $2 \mathrm{i}$ LIF DOXiPSC-derived reconstructed embryos. After the SCNT experiments we discovered that the $2 \mathrm{i}$ LIF DOX-iPSC had acquired an additional x-chromosome. Similarly, SCNT based on Pl-iPSCs resulted in blastocyst rates $(10.1 \% \mathrm{n}=148)$ and quality (Figure 2G) comparable to the $2 \mathrm{i}$ LIF DOX-iPSC. The 2i FGF Pl-iPSCs were karyotypically normal, but due to the low blastocyst rate and quality no attempts were made to transfer the 2i FGF Pl-iPSC-derived embryos to surrogate sows.

\subsection{Embryo transfer and birth of piglets}


256 Only reconstructed embryos derived from the WL-PNFs and EGCs were used for transfer. A total 257 of 126 WL-PNF-derived embryos were transferred to two surrogate sows (58 and 68 embryos per sow, respectively). One sow became pregnant and birth was induced at day 117 with $1 \mathrm{ml}$ Estrumat vet (MSD Animal Health). One piglet was born vaginally while three were delivered by cesarean section. The four piglets corresponds to a cloning efficiency of $3.2 \%$ (live born piglets/embryos transferred; Figure 3A, C). All piglets were alive at birth and all expressed the Venus (green) fluorescent protein (Figure 3B-D). The piglet that was born naturally, exhibited macroglossia and bilateral cryptorchidism and died within two hours after birth. The other three piglets, delivered via caesarian section, had mild macroglossia and one had bilateral cryptorchidism and was euthanized after 6 hours. Necropsy of the piglets showed that all tissues expressed the Venus protein (for examples see Figure 3B, D). A total of 200 EGC-derived embryos were transferred to two surrogate sows (76 and 124 embryos per sow, respectively). Both sows became pregnant and a total of eight liveborn and four stillborn piglets, as well as four mummified fetuses were delivered vaginally. The 12 piglets delivered correspond to a cloning efficiency of $4.0 \%$. Three piglets died within a few days after delivery and 4 were euthanized shortly after birth due to failure to thrive and, in two cases, paraplegia and tetraplegia, leaving only one surviving piglet (Figure 4A). Necropsy of the dead piglets revealed immature organs, septal defects in the heart, and in the piglet with tetraplegia, cerebellar hypoplasia was observed (Figure 4B). Septal defects in the heart are quite common in the Yucatan breed, and we have seen this phenomenon previously when cloning from Yucatan fibroblasts (Schmidt et al., 2015). The transgene itself is unlikely to be the underlying cause of these defects as we have previously cloned Yucatan pigs carrying a mutant form of the PCSK9 gene, or other transgenes, with success and without observing as severe malformations as observed in the pigs derived from EGCs. Apart from one, all EGC-derived piglets were found to comprise the WT-PCSK9-FLAG transgene by PCR analysis (Figure 4C). Southern blot analysis performed on genomic DNA from all EGC-derived piglets revealed variable copy number and different integration sites of the 283 Southern blot analysis of genomic DNA isolated from the PCSK9-negative piglet indicated random integration of the entire vector via a breakage point in the PCSK9 part of the vector rather than integration of the transposon by transposition thereby impairing the PCSK9-specific PCR (Figure 1 , piglet 6 , supplementary data). In the surviving piglet, two copies of the transgene were detected by Southern blotting (Figure 1, piglet 2, supplementary data). The piglet was found to be 
288 karyotypically normal (Figure 4D) and, as expected, it exhibited abundant liver-specific expression of human WT-PCSK9-FLAG (Figure 4E). This founder animal was subsequently bred with a wild type Yucatan boar resulting in normal offspring. Southern blot analysis of genomic DNA isolated

291 from the founder animal and its offspring validated transmission of the WT-PCSK9-FLAG transgene cassette to all offspring (data not shown).

\section{Discussion}

Our experiments did not sustain the hypothesis that increased cell potency is positively correlated to the ability to sustain embryonic development after SCNT. We found that cells of the expected highest potency (2i LIF DOX-iPSC and 2i FGF Pl-iPSC) resulted in the lowest blastocyst rates as well as the poorest embryo quality when compared with cells of medium (EGCs) and low potency (WL-PNFs and G-PEFs). Futhermore, we did not see a higher ability of the $2 \mathrm{i}$ LIF DOX-iPSC to sustain embryo development over the 2i FGF Pl-iPSC.

302 Possible explanations for the fact that our piPSC lines have low low capacity to sustain embryo development after SCNT can be that the piPSCs are potentially not fully reprogrammed, not

304 cultured under the right conditions (Hall and Hyttel, 2014), exhibit failure of silencing of the transgenes (Takahashi and Yamanaka, 2006), have undergone insertional mutagenesis caused by lentiviral integration of the transgenes (Higuchi et al., 2015) or because it is difficult to force iPSCs into the G1 or G2 phase making them less compatible for SCNT (Yuan et al., 2014); however this last problem should also affect the EGC. Karyotyping results revealed that the 2i FGF PL-iPSC had a normal karyotype, but the 2i LIF DOX-iPSC had acquired an additional X-chromosome. This can potentially explain the low clonogenicity observed with the 2i LIF DOX-iPSC, but since Du and coworkers (Du et al., 2015) reported a similarly low clonogenicity of a piPSC line with normal karyotype and cultured in $2 \mathrm{i}$ LIF conditions, this was not pursued any further.

314 The SCNT experiments with the EGCs showed that it was possible to produce blastocysts at a 315 relatively high rate and quality allowing for transfer and generation of live offspring even though 316 only one out of eight born piglets survived. Thus, we can conclude from our SCNT and embryo 317 transfer experiments using transgenic EGCs and fibroblasts that generation of transgenic pigs is 318 possible from both cell types. Nevertheless, there is no apparent advantage using EGCs over 319 fibroblasts apart from an enhanced in vitro live span of the EGCs. In fact, we observed more severe 
320 developmental defects in the cloned piglets derived from EGCs than when using comparable 321 transgenic Yucatan fibroblasts (see (Schmidt et al., 2015) for a thorough description on

322 developmental defects in piglets made by SCNT). Ahn and coworkers (Ahn et al., 2010) also 323 succeed in creating CD59-transgenic piglets using porcine EGC for SCNT. They transferred 1980 324 reconstructed embryos to 10 recipient sows resulting in 5 pregnancies and 15 piglets of which 5 325 died within the first 24 hours (Ahn et al., 2010) and thus also indicating that the EGCs do not have 326 the same clonogenicity as murine embryonic cells (Oback, 2009). Finally, creating and maintaining 327 porcine EGCs are laborious processes that require special technical skills (Petkov et al., 2011)

329 It should be mentioned that the data for this paper is not collected as part of an experimental set up 330 made to explore the correlation between cell differentiation potency and clonogenicity. It is 331 collected from experiments made to optimize SCNT by using different cell lines. The donor cells 332 used are not isogenic and thus do not comprise an easy comparable background, and factors such as 333 gender, pig breed and genetic modification may affect the clonegenicity of a cell line (Schmidt et 334 al., 2015). Thus, simply comparing cells with regard to differentiation potency should be done with 335 some caution. Despite the lack of a systematic evaluation of only isogenic cell types, however, the 336 data presented clearly shows that reprogramming of the WL-PNFs to 2i LIF DOX-iPSC and the G337 PEFs to $2 \mathrm{i}$ FGF Pl-iPSC in both cases resulted in lower clonogenicity. It also indicate that there is 338 only minor differences in clonogenicity in iPS cells cultured with LIF and FGF and also among 339 cells reprogrammed with an integrating lentiviral construct or a non-integrating plasmid. We 340 therefore believe that this dataset can contribute to the debate regarding the dogma that pluripotent 341 stem cells can increase the SCNT efficiency in comparison to somatic cells as well a to the debate 342 concerning whether it is possible to transfer the effect of the FGF conditions vs the 2i LIF 343 conditions from murine pluripotent stem cells (Nichols and Smith, 2009) to the porcine pluripotent 344 stem cells

345 In conclusion, in our experiments neither EGC nor iPSC resulted in higher SCNT efficiency 346 compared to the tested fibroblast lines, and similar low efficient clonogenicity was observed when comparing DOX-iPSC cultured with 2i LIF and Pl-iPSC cultured with 2i FGF. 
The authors would like to thank Trine Skov Petersen, Christian Knudsen, Lisa Maria Røge, Tina

Christoffersen, Annedorte Valentin Roed, Janne Adamsen, Ruth Kristensen, Anette M Pedersen and

Klaus Villemoes for excellent technical assistance.

Funding for this research was provided by:

The Danish Council for Independent Research, Natural Sciences (FNU), grant number: 11-106627;

The Villum Kann Rasmussen Foundation; The US Department of Agriculture Grant number: 201167015-30688; The Danish National Advanced Technology Foundation, grant numbers: 047-2011-1 and 274-05-0535. Göttingen minipig embryos were kindly provided by Ellegaard Göttingen

Minipigs.

\section{Author Disclosure Statement}

The authors declare that they have no competing financial interests.

\section{References}

Ahn, K.S., Won, J.Y., Park, J.K., Sorrell, A.M., Heo, S.Y., Kang, J.H., Woo, J.S., Choi, B.H., Chang, W.K., Shim, H., 2010. Production of human CD59-transgenic pigs by embryonic germ cell nuclear transfer. Biochem. Biophys. Res. Commun. 400, 667-672.

Al-Mashhadi, R.H., Sorensen, C.B., Kragh, P.M., Christoffersen, C., Mortensen, M.B., Tolbod, L.P., Thim, T., Du, Y., Li, J., Liu, Y., Moldt, B., Schmidt, M., Vajta, G., Larsen, T., Purup, S., Bolund, L., Nielsen, L.B., Callesen, H., Falk, E., Mikkelsen, J.G., Bentzon, J.F., 2013. Familial hypercholesterolemia and atherosclerosis in cloned minipigs created by DNA transposition of a human PCSK9 gain-of-function mutant. Sci. Transl. Med. 5, 166ra1.

Bassols, A., Costa, C., Eckersall, P.D., Osada, J., Sabria, J., Tibau, J., 2014. The pig as an animal model for human pathologies: A proteomics perspective. Proteomics.Clinical Appl. 8, 715731.

Callesen, H., Liu, Y., Pedersen, H.S., Li, R., Schmidt, M., 2014. Increasing efficiency in production of cloned piglets. Cell. Reprogram. 16, 407-410.

Cao, S., Han, J., Wu, J., Li, Q., Liu, S., Zhang, W., Pei, Y., Ruan, X., Liu, Z., Wang, X., Lim, B., Li, N., 2014. Specific gene-regulation networks during the pre-implantation development of the pig embryo as revealed by deep sequencing. BMC Genomics 15, 4.

Cheng, D., Guo, Y., Li, Z., Liu, Y., Gao, X., Gao, Y., Cheng, X., Hu, J., Wang, H., 2012. Porcine induced pluripotent stem cells require LIF and maintain their developmental potential in early stage of embryos. PLoS One 7, e51778.

Du, X., Feng, T., Yu, D., Wu, Y., Zou, H., Ma, S., Feng, C., Huang, Y., Ouyang, H., Hu, X., Pan, D., Li, N., Wu, S., 2015. Barriers for Deriving Transgene-Free Pig iPS Cells with Episomal Vectors. Stem Cells 33, 3228-3238.

Esteban, M.A., Xu, J., Yang, J., Peng, M., Qin, D., Li, W., Jiang, Z., Chen, J., Deng, K., Zhong, M., Cai, J., Lai, L., Pei, D., 2009. Generation of induced pluripotent stem cell lines from Tibetan miniature pig. J. Biol. Chem. 284, 17634-17640.

Ezashi, T., Telugu, B.P., Alexenko, A.P., Sachdev, S., Sinha, S., Roberts, R.M., 2009. Derivation of 
induced pluripotent stem cells from pig somatic cells. Proc. Natl. Acad. Sci. U. S. A. 106, 10993-10998.

Fan, N., Chen, J., Shang, Z., Dou, H., Ji, G., Zou, Q., Wu, L., He, L., Wang, F., Liu, K., Liu, N., Han, J., Zhou, Q., Pan, D., Yang, D., Zhao, B., Ouyang, Z., Liu, Z., Zhao, Y., Lin, L., Zhong, C., Wang, Q., Wang, S., Xu, Y., Luan, J., Liang, Y., Yang, Z., Li, J., Lu, C., Vajta, G., Li, Z., Ouyang, H., Wang, H., Wang, Y., Yang, Y., Liu, Z., Wei, H., Luan, Z., Esteban, M.A., Deng, H., Yang, H., Pei, D., Li, N., Pei, G., Liu, L., Du, Y., Xiao, L., Lai, L., 2013. Piglets cloned from induced pluripotent stem cells. Cell Res. 23, 162-166.

Fujishiro, S.H., Nakano, K., Mizukami, Y., Azami, T., Arai, Y., Matsunari, H., Ishino, R., Nishimura, T., Watanabe, M., Abe, T., Furukawa, Y., Umeyama, K., Yamanaka, S., Ema, M., Nagashima, H., Hanazono, Y., 2013. Generation of naive-like porcine-induced pluripotent stem cells capable of contributing to embryonic and fetal development. Stem Cells Dev. 22, 473-482.

Garrels, W., Mates, L., Holler, S., Dalda, A., Taylor, U., Petersen, B., Niemann, H., Izsvak, Z., Ivics, Z., Kues, W.A., 2011. Germline transgenic pigs by Sleeping Beauty transposition in porcine zygotes and targeted integration in the pig genome. PLoS One 6, e23573.

Hall, V.J., Hyttel, P., 2014. Breaking down pluripotency in the porcine embryo reveals both a premature and reticent stem cell state in the inner cell mass and unique expression profiles of the naive and primed stem cell states. Stem Cells Dev. 23, 2030-2045.

Higuchi, A., Ling, Q.D., Kumar, S.S., Munusamy, M.A., Alarfaj, A.A., Chang, Y., Kao, S.H., Lin, K.C., Wang, H.C., Umezawa, A., 2015. Generation of pluripotent stem cells without the use of genetic material. Lab. Invest. 95, 26-42.

Kawaguchi, T., Tsukiyama, T., Kimura, K., Matsuyama, S., Minami, N., Yamada, M., Imai, H., 2015. Generation of Naïve Bovine Induced Pluripotent Stem Cells Using PiggyBac

Transposition of Doxycycline-Inducible Transcription Factors. PLoS One 10, e0135403. doi:10.1371/journal.pone.0135403

Lee, K., Redel, B.K., Spate, L., Teson, J., Brown, A.N., Park, K.W., Walters, E., Samuel, M., Murphy, C.N., Prather, R.S., 2013. Piglets produced from cloned blastocysts cultured in vitro with GM-CSF. Mol. Reprod. Dev. 80, 145-154.

Lelovas, P.P., Kostomitsopoulos, N.G., Xanthos, T.T., 2014. A comparative anatomic and physiologic overview of the porcine heart. J. Am. Assoc. Lab. Anim. Sci. 53, 432-438.

Li, J., Li, R., Liu, Y., Villemoes, K., Purup, S., Callesen, H., 2013. Developmental kinetics of pig embryos by parthenogenetic activation or by handmade cloning. Reprod. Domest. Anim. 48, 866-873.

Liu, Y., Li, J., Lovendahl, P., Schmidt, M., Larsen, K., Callesen, H., 2014. In vitro manipulation techniques of porcine embryos: a meta-analysis related to transfers, pregnancies and piglets. Reprod. Fertil. Dev.

Nethercott, H.E., Brick, D.J., Schwartz, P.H., 2011. Derivation of induced pluripotent stem cells by lentiviral transduction. Methods Mol. Biol. 767, 67-85.

Nichols, J., Smith, A., 2009. Naive and primed pluripotent states. Cell Stem Cell 4, 487-492.

Oback, B., 2009. Cloning from stem cells: different lineages, different species, same story. Reprod. Fertil. Dev. 21, 83-94.

Okita, K., Matsumura, Y., Sato, Y., Okada, A., Morizane, A., Okamoto, S., Hong, H., Nakagawa, M., Tanabe, K., Tezuka, K., Shibata, T., Kunisada, T., Takahashi, M., Takahashi, J., Saji, H., Yamanaka, S., 2011. A more efficient method to generate integration-free human iPS cells. Nat. Methods 8, 409-412.

Onishi, A., Iwamoto, M., Akita, T., Mikawa, S., Takeda, K., Awata, T., Hanada, H., Perry, A.C., 2000. Pig cloning by microinjection of fetal fibroblast nuclei. Science 289, 1188-1190. 
Petkov, S.G., Marks, H., Klein, T., Garcia, R.S., Gao, Y., Stunnenberg, H., Hyttel, P., 2011. In vitro culture and characterization of putative porcine embryonic germ cells derived from domestic breeds and Yucatan mini pig embryos at Days 20-24 of gestation. Stem Cell Res. 6, 226-237.

Polejaeva, I.A., Chen, S.H., Vaught, T.D., Page, R.L., Mullins, J., Ball, S., Dai, Y., Boone, J., Walker, S., Ayares, D.L., Colman, A., Campbell, K.H., 2000. Cloned pigs produced by nuclear transfer from adult somatic cells. Nature 407, 86-90.

Schmidt, M., Kragh, P.M., Li, J., Du, Y., Lin, L., Liu, Y., Bogh, I.B., Winther, K.D., Vajta, G., Callesen, H., 2010. Pregnancies and piglets from large white sow recipients after two transfer methods of cloned and transgenic embryos of different pig breeds. Theriogenology 74, 12331240.

Schmidt, M., Winter, K.D., Dantzer, V., Li, J., Kragh, P.M., Du, Y., Lin, L., Liu, Y., Vajta, G., Sangild, P.T., Callesen, H., Agerholm, J.S., 2011. Maternal endometrial oedema may increase perinatal mortality of cloned and transgenic piglets. Reprod. Fertil. Dev. 23, 645-653.

Schmidt, M., Winther, K.D., Secher, J.O., Callesen, H., 2015. Postmortem findings in cloned and transgenic piglets dead before weaning. Theriogenology 84, 1014-1023.

Secher, J.O., Freude, K.K., Li, R., Callesen, H., 2015. Optimization of Three-Dimensional Imaging on In Vitro Produced Porcine Blastocysts and Chimeras for Stem Cell Testing: A Technology Report. Stem Cells Dev.

Secher, J.O., Ceylan, A., Mazzoni, G., Mashayekhi, K., Li, T., Muenthaisong, S., Nielsen, T.T., Li, D., Li, S., Petkov, S., Cirera, S., Luo, Y., Thombs, L., Kadarmideen, H.N., Dinnyes, A., Bolund, L., Roelen, B.A.J., Schmidt, M., Callesen, H., Hyttel, P., Freude, K.K., 2017. Systematic in vitro and in vivo characterization of Leukemia-inhibiting factor- and Fibroblast growth factor-derived porcine induced pluripotent stem cells. Mol. Reprod. Dev. doi:10.1002/mrd.22771

Takahashi, K., Yamanaka, S., 2006. Induction of pluripotent stem cells from mouse embryonic and adult fibroblast cultures by defined factors. Cell 126, 663-676.

Telugu, B.P., Ezashi, T., Roberts, R.M., 2010. The promise of stem cell research in pigs and other ungulate species. Stem Cell Rev. 6, 31-41.

Telugu, B.P., Ezashi, T., Sinha, S., Alexenko, A.P., Spate, L., Prather, R.S., Roberts, R.M., 2011. Leukemia inhibitory factor (LIF)-dependent, pluripotent stem cells established from inner cell mass of porcine embryos. J. Biol. Chem. 286, 28948-28953.

Wilmut, I., Schnieke, A.E., McWhir, J., Kind, A.J., Campbell, K.H., 1997. Viable offspring derived from fetal and adult mammalian cells. Nature 385, 810-813.

Wu, Z., Chen, J., Ren, J., Bao, L., Liao, J., Cui, C., Rao, L., Li, H., Gu, Y., Dai, H., Zhu, H., Teng, X., Cheng, L., Xiao, L., 2009. Generation of pig induced pluripotent stem cells with a druginducible system. J. Mol. Cell Biol. 1, 46-54.

Yuan, Y., Lee, K., Park, K.-W., Spate, L.D., Prather, R.S., Wells, K.D., Roberts, R.M., 2014. Cell cycle synchronization of leukemia inhibitory factor (LIF)-dependent porcine-induced pluripotent stem cells and the generation of cloned embryos. Cell Cycle 13, 1265-76. doi:10.4161/cc. 28176

\section{Figure and table legends}

Figure 1 A. Isolation of G-PEFs used to generate 2i FGF pl-iPSC. Both G-PEFs and 2i FGF pl-iPSC were used for Handmade Cloning; B. Isolation of EGC which was genetically modified and used for Handmade Cloning; C. Isolation of (Venus) WL-PNFs used to generate 2i LIF DOX-iPSC. Both WL-PNF and 2i LIF DOX-iPSC were used for Handmade cloning; D. Overview of cells used for SCNT; E. Blastocyst percentage from the different cell lines 
presented in a g-plot. * Other refers to reconstructed embryos that did not develop to blastocyst. Embryo transfer is abbreviated ET in the figure.

Table 1. A. Output from Procfreq SAS 5.1. showing number of replicates and calculations for blastocyst percentages; B. Results from Proclogistic from SAS 5.1. showing odds ratio for comparison of cell lines.

Figure 2. A \& F 6 days old SCNT embryos from WL-PNF; B, 6 days old SCNT embryos from G-PEFs; G, 6 days old SCNT embryos from 2i FGF Pl-iPSCs; C \& H, 6 days old SCNT embryos from 2i LIF DOX-iPSC cultured in PZM3 (Group 1); D \& I, 6 days old SCNT embryos from 2i LIF DOX-iPSC cultured in PZM3 with 10\% FCS (Group 2); E \& J, 6 days old SCNT embryos from 2i LIF DOX-iPSC cultured in PZM3 with $2 \mu \mathrm{g} / \mathrm{ml}$ doxycycline (Group 3). Arrows indicate blastomers in developmental arrest. Scale bars are $100 \mu \mathrm{m}$.

Figure 3. Pictures of the newborn SCNT piglets from WL-PNF. A \& C, taken in day light; B \& D, taken in ultraviolet light; C \& D, piglet with macroglosia and green fluorescent eye (arrow) in daylight.

Figure 4: A, Healthy cloned SCNT offspring from EGC; B, Necropsy of euthanized piglet with tetraplegia showing hypoplasia of cerebellum; C, PCSK9-specific PCR performed on genomic DNA isolated from the eight SCNT piglets from EGC (id 1-8); D, Karyotype of the surviving SCNT EGC healthy female founder animal; E, qPCR showing liverspecific expression of PCSK9 in the surviving SCNT EGC founder animal.

\section{Supplementary data}

Figure 1. Southern blot detection of transgene integrations in the eight SCNT piglets from EGCs. Both short (panel A) and longer exposure of the blot (panel B) are shown due to high transgene copy number in one of the piglets (piglet 1). The transgene was visualized using a probe recognizing a 1-kb sequence in the puromycin resistance gene cassette. The id numbers (1-8) of the piglets are indicated above the lanes. Plasmid copy number controls were made by spiking genomic DNA from a WT Yucatan mini-pig with different amounts of the pSBT/ApoEHCR-hAATp-WT-PCSK9FLAG plasmid for detection of $1(\mathrm{C} 1)$ and 10 copies (C10) of the transgene. Genomic DNA from a WT Yucatan minipig was used as negative control (WT). All eight piglets were transgenic. The approx. $5 \mathrm{~kb}$ band observed for piglet 6 , however, is indicative of random integration of the vector rather than transposition which may have occurred via a breakage point within the PCSK9 part of the vector. Arrows indicate the two transgene integrations in the surviving founder animal (piglet 2). 


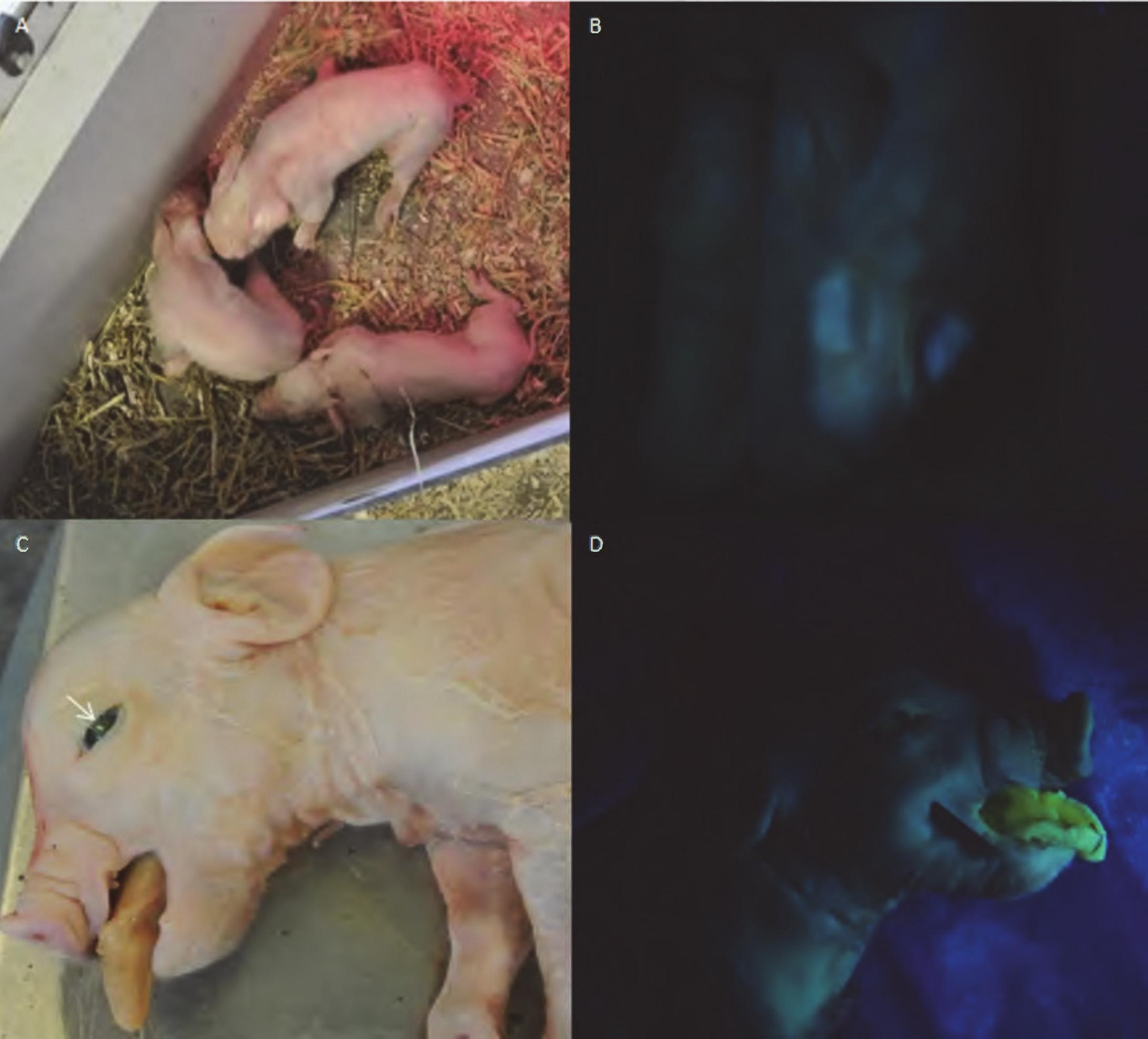




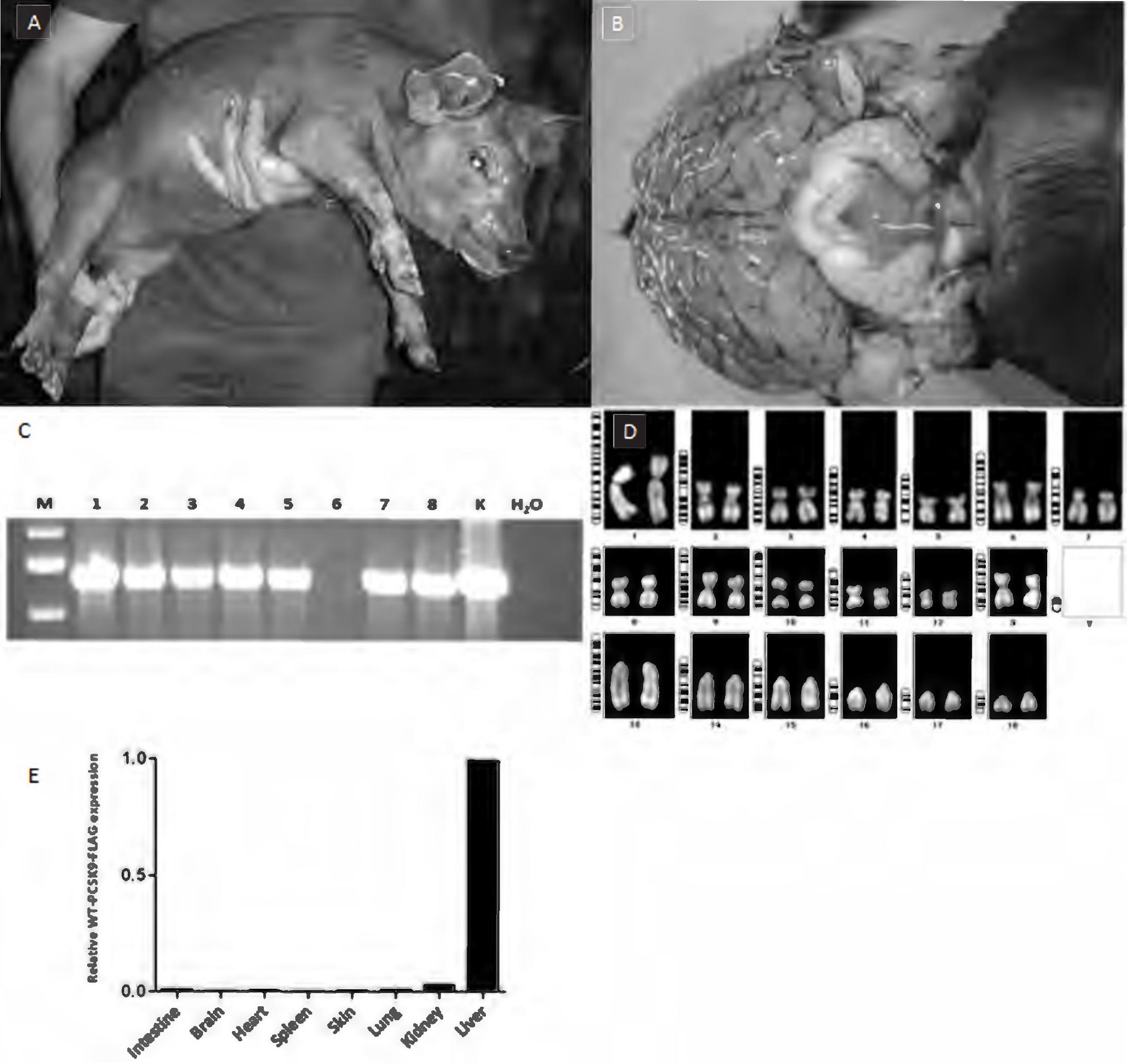




\begin{tabular}{l|l|l} 
A \\
$\begin{array}{l}\text { Cell type/No. } \\
\text { of replicates }\end{array}$ & $\begin{array}{l}\text { Blastocyst/reconstructed } \\
\text { embryos }\end{array}$ & Blastocyst rate \\
\hline WL-PNF/3 & $144 / 392$ & $36.7 \%$ \\
\hline $\begin{array}{l}2 i \text { LIF DOX- } \\
\text { iPSC in PZM/5 }\end{array}$ & $13 / 122$ & $10,7 \%$ \\
\hline $\begin{array}{l}2 i \text { LIF DOX- } \\
\text { iPSC in PZM } \\
\text { with FCS/3 }\end{array}$ & $13 / 63$ & 20,6 \\
\hline $\begin{array}{l}2 i \text { LIF DOX- } \\
\text { iPSC in PZM } \\
\text { with DOX/1 }\end{array}$ & $4 / 18$ & \\
\hline $\begin{array}{l}2 i \text { LIF DOX- } \\
\text { iPSC/5 }\end{array}$ & $30 / 203^{*}$ & 22,2 \\
\hline G-PEF/4 & $48 / 190$ & $14.7 \%$ \\
\hline $2 i$ FGF PI- & $15 / 148$ & $25.2 \%$ \\
\hline iPSC/3 & & $10.1 \%$ \\
\hline EGC/2 & $51 / 148$ & $34.5 \%$ \\
\hline B & & \\
\hline
\end{tabular}

B

\begin{tabular}{|c|c|c|}
\hline $\begin{array}{l}\text { Cell type vs cell } \\
\text { type }\end{array}$ & $\begin{array}{l}\mathrm{Pr}>\mathrm{ChiSq} \\
\text { (proclogistic } \\
\text { SAS) }\end{array}$ & $\begin{array}{l}\text { Odds ratio (confidence } \\
\text { limits) for blastocyst rate }\end{array}$ \\
\hline $\begin{array}{l}2 i \text { LIF DOX iPSC vs } \\
\text { WL-PNF }\end{array}$ & $P<0.0001$ & $0.205(0.112 ; 0.378)$ \\
\hline WL-PNF vs G-PEF & $P=0.006$ & $1.718(1.167 ; 2.528)$ \\
\hline $\begin{array}{l}\text { 2i LIF DOX-iPSC vs } \\
\text { 2i FGF PI-iPSC }\end{array}$ & $P=0.889$ & $0.946(0.432 ; 2.073)$ \\
\hline $\begin{array}{l}2 i \text { LIF DOX-iPSC } \\
\text { without FCS vs } 2 i \\
\text { LIF DOX-iPSC with } \\
\text { FCS }\end{array}$ & $P=0.069$ & $0.459(0.198 ; 1.061)$ \\
\hline $\begin{array}{l}2 i \text { LIF DOX-iPSC } \\
\text { without DOX vs } 2 \mathrm{i} \\
\text { LIF DOX-iPSC with } \\
\text { DOX }\end{array}$ & $P=0.171$ & $0.418(0.119 ; 1.061)$ \\
\hline EGC vs 2i FGF PI- & $P<0.001$ & $4.61(2.45 ; 8.70)$ \\
\hline
\end{tabular}




\section{iPSC \\ EGC vs WL-PNF \\ $\mathrm{P}=0.587$ \\ $1.12(0.771 ; 1.658)$}

${ }^{*}$ Pool of 2i LIF DOX-iPSC with and without doxycycline and fetal calf serum. ${ }^{* *}$ WL-PNF and 2i FGF PI-iPSC have been used as intercept but the model has not been extended to calculate the odds ratio for all the cell lines, as the model showed no difference between the remaining cell lines. 